\title{
Peculiarities of calcium-phosphorus homeostasis of the oral fluid in young patients with primary hypothyroidism, having generalized periodontitis
}

\author{
Repetska Oksana Mykolaivna \\ Ivano-Frankivsk National Medical University, Ivano-Frankivsk, Ukraine
}

\begin{abstract}
The problem of hormonal imbalance in periodontal diseases, as well as the importance of endocrine diseases in its development, plays a significant role. In the professional literature there are data that thyroid disease is a risk factor for the occurrence and further progression of periodontal diseases. The processes of bone modeling and remodeling and its mineralization are closely related to calcium metabolism. The biochemical manifestation of osteoporotic processes in the alveolar bone in generalized periodontitis is a violation of calcium-phosphorus homeostasis against the background of altered markers of bone metabolism. The aim of our investigation was to study the calcium-phosphorus homeostasis of the oral fluid in patients with primary hypothyroidism and persons without endocrine pathology, with generalized periodontitis. Since in the structure of periodontal diseases in the study groups generalized periodontitis of the initial-I and II degree had the largest percentage, biochemical studies were performed in this sample of patients. The first group included 50 people with generalized periodontitis against the background of primary hypothyroidism: 25 patients - with generalized periodontitis of the initial-I degree and 25 patients with generalized periodontitis of the II degree of development. The second group included 50 patients with generalized periodontitis without endocrinological pathology (25 patients with GP of the initial-I degree and 25 patients with GP of the II degree of development). In young people with generalized periodontitis, against the background of hypothyroidism, there were observed negative changes in calcium-phosphorus homeostasis of the oral fluid: there was a tendency to the decrease of calcium content in the oral fluid with the development of inflammatory-dystrophic process in the periodontium, on the contrary, phosphorus level has increased. Negative dynamics of calcium-phosphorus molar coefficient was found in patients with hypofunction of the thyroid gland. Thus, the analysis of research results shows that in young people with periodontal diseases and hypothyroidism there is a pathological change in metabolic processes, increased excretion of mineral components, especially calcium. Periodontal pathology against the background of hypothyroidism is characterized by the development of a chronic inflammatory process together with a pronounced decrease in calcium-phosphorus homeostasis of the oral fluid.
\end{abstract}

Keywords: hypothyroidism, young age, generalized periodontitis, oral fluid, calcium-phosphorus homeostasis

\section{INTRODUCTION}

The number of organs and systems responding to thyroid hormones includes the issues of thyroidology into the field of interest of various medical specialties, and the growing incidence of thyroid diseases among the population brings these problems to the forefront of modern endocrinology. Currently, close attention is drawn to thyroid diseases associated with endemic foci of iodine deficiency in soil and water [1,2]. Most often, the pathology of the 
thyroid system is accompanied by hypothyroidism syndrome, caused by insufficient production of thyroid hormones and peripheral disorders of their action [3].

The problem of hormonal imbalance in periodontal diseases, as well as the importance of endocrine diseases in its development, has a significant role. It is known that the state and interaction of the main regulatory systems (nervous, endocrine and immune ones) determines the homeostasis of the organism and its reaction to any pathogenic influence. In the professional literature there are data that thyroid diseases are some risk factors for the occurrence and further progression of periodontal diseases [4-8].

The processes of modeling and remodeling of bone and its mineralization are closely related to calcium metabolism. The biochemical manifestation of osteoporotic processes in the alveolar bone in generalized periodontitis is a violation of calcium-phosphorus homeostasis against the background of altered markers of bone metabolism [9$11]$.

It is known that the elemental composition of the organism can be judged with sufficient confidence according to the content of individual macro- and micronutrients in various biosubstrates: blood, urine, oral fluid. The study of oral fluid as a biological environment that washes teeth and the mucous membrane of the oral cavity is of particular interest to dentists. Oral fluid belongs to the integral environmental media of the human body, therefore, both metabolic processes affect its composition and components of oral fluid have local and systemic effects, which allows us to consider it an important factor in maintaining human health [12].

\section{AIM}

The aim of our study was to investigate the calcium-phosphorus homeostasis of the oral fluid in patients with primary hypothyroidism and persons without endocrine pathology, with generalized periodontitis.

\section{MATERIALS AND METHODS}

Since in the structure of periodontal diseases in study groups generalized periodontitis of the initial-I and II degree of development had the largest percentage, biochemical studies were performed in this sample of patients. The first group included 50 patients with generalized periodontitis against the background of primary hypothyroidism: 25 patients - with generalized periodontitis of the initial-I degree and 25 patients with generalized periodontitis of the II degree of development. The second group included 50 patients with generalized periodontitis without endocrinological pathology (25 patients with GP of the initial-I degree and 25 patients with GP of the II degree of development).

Determination of the amount of calcium in the oral fluid was performed using a kit for the determination of calcium in biological fluids (research-production company Simko, Ukraine). The principle of the method action is based on the ability of calcium to form a blue complex in a neutral medium with the stain Arsenazo III measured photocolorimetrically (photocolorimeter KFK-2MP) at a wavelength of 590-650 nm.

Quantitative determination of phosphorus content in the oral fluid was performed with atomic-absorption spectrophotometry using standard sets of reagents and control sera Filo-Norm, Filo-Pat made by company SpineLab (Ukraine) on the atomic-sorption spectrophotometer Saturn-3, equipped with an electrothermal atomizer Graphite-2 according to the standard method described in the factory operating instructions.

To objectively assess the reliability of the research results, statistical processing of the obtained data was performed with the help of conventional methods of variation statistics using a Pentium II personal computer and statistical software package Statgraphic 2.3 and Microsoft Excel 2000. Statistical processing of the results obtained was performed by calculating the arithmetic mean (M), standard deviation (б), mean error (m). The degree of reliability (p) of the obtained results was determined by t-criterion.

\section{RESULTS}

The results of studies of calcium-phosphorus homeostasis of the patients' oral fluid in both observation groups are represented in Table 1.

The average value of calcium content in the oral fluid of young people with generalized periodontitis against the background of hypothyroidism was $1.29 \pm 0.28 \mathrm{mmol} / \mathrm{l}$ and was within the normative values for this index, but was 1.2-fold lower than that of persons without endocrine pathology, $\mathrm{p}<0.01$.

With the development of the inflammatory-dystrophic process in the periodontium, a decrease in the calcium content in the oral fluid of group I patients - 1.09-fold $(1.23 \pm 0.31 \mathrm{mmol} / \mathrm{l}, \mathrm{p}<$ 0.01 ) was observed.

In generalized periodontitis of the initial-I degree of development in patients of group I the concentration index of inorganic phosphorus in the oral fluid was $3.87 \pm 0.54 \mathrm{mmol} / \mathrm{l}$. In generalized periodontitis of the II degree of development, an increase of the phosphorus content in the oral fluid was observed: the digital value of $4.60 \pm 0.62 \mathrm{mmol} / \mathrm{l}$ approached the upper margin of normal for this indicator. The average value of phosphorus in the oral 
TABLE 1. Indices of calcium-phosphorus homeostasis of the oral fluid in the study groups

\begin{tabular}{|c|c|c|c|c|}
\hline \multirow[b]{2}{*}{ Index } & \multicolumn{2}{|c|}{ I group $(n=50)$} & \multicolumn{2}{|c|}{ II group $(n=50)$} \\
\hline & $\begin{array}{c}\text { GP of the initial-I } \\
\text { degree }\end{array}$ & $\begin{array}{l}\text { GP of the } \\
\text { II degree }\end{array}$ & $\begin{array}{l}\text { GP of the initial } \\
\text { I degree }\end{array}$ & $\begin{array}{l}\text { GP of the } \\
\text { II degree }\end{array}$ \\
\hline Concentration of $\mathrm{Ca}(\mathrm{mmol} / \mathrm{l})$ & $1,35 \pm 0,25 * \bullet$ & $1,23 \pm 0,31^{*}$ & $1,46 \pm 0,34 \bullet$ & $1,52 \pm 0,37$ \\
\hline Concentration of $\mathrm{P}(\mathrm{mmol} / \mathrm{l})$ & $3,87 \pm 0,54^{* \bullet}$ & $4,60 \pm 0,62^{*}$ & $2,52 \pm 0,43^{\bullet}$ & $2,47 \pm 0,40$ \\
\hline $\begin{array}{l}\text { Coefficient of } \\
\mathrm{Ca} / \mathrm{P} \text { (un) }\end{array}$ & $0,34 \pm 0,07 * \bullet$ & $0,27 \pm 0,06 *$ & $0,58 \pm 0,11^{\bullet \bullet}$ & $0,61 \pm 0,12$ \\
\hline
\end{tabular}

${ }^{*} p<0.01$ - significant difference of values in relation to the data of group II;

$\bullet p 1<0.01$ - a significant difference in values between the indices of group I

$\bullet p 2<0.01-a$ significant difference of values between indices of group II

fluid of patients with generalized periodontitis against the background of reduced thyroid function was $4.24 \pm 0.58 \mathrm{mmol} / \mathrm{l}$ and was 1.7 -fold higher than similar in the group of persons without endocrinological pathology, $\mathrm{p}<0.01$.

Normally, the calcium-phosphorus ratio in the oral fluid is in the range of 0.5-1.2 units. With the progression of the inflammatory-dystrophic process in the periodontium of patients in group I, a decrease in the calcium-phosphorus coefficient from $0.34 \pm 0.07$ units up to $0.27 \pm 0.06$ units was observed. In patients with hypothyroidism, the average value of this index was $0.30 \pm 0.07$ units, which was less than the lower limit of the reference values and 2-fold less than the index of group II, $\mathrm{p}<0.01$ (Fig. 1).

In the study group II, the opposite dynamics of the content of mineral elements in the oral fluid was observed (Fig. 2). The level of calcium in the oral fluid increased from the digital indicator of $1.46 \pm 0.34 \mathrm{mmol} / \mathrm{l}$ in $\mathrm{GP}$ of the initial-I degree to $1.52 \pm 0.37 \mathrm{mmol} / \mathrm{l}$ in GP of the II degree of development, $p<0.01$. However, the concentration index of inorganic phosphorus in the oral fluid of persons not burdened with endocrine pathology, tended to decrease with the development of the pathological process in the periodontium $(2.52 \pm 0.43 \mathrm{mmol} / \mathrm{l}$ and $2.47 \pm 0.40 \mathrm{mmol} / \mathrm{l}, \mathrm{p}<0.01$ ).

Calcium-phosphorus coefficient in persons of group II increased from $0.58 \pm 0.11$ units in GP of the I degree to $0.61 \pm 0.12$ units in GP of the II degree, $\mathrm{p}<$ 0.01 . The average value of calcium-phosphorus ratio in the oral fluid of persons not burdened with endocrinological pathology $(0.59 \pm 0.12$ units $)$ was within the normative values and was 2-fold higher than that of patients with the primary hypothyroidism, $\mathrm{p}<0.01$.

\section{DISCUSSION}

The results of our studies objectified that the content of calcium and phosphorus in the oral fluid are closely interrelated.

Thus, in young people with generalized periodontitis, against the background of hypothyroidism, negative changes in calcium-phosphorus homeostasis of the oral fluid were observed: there was found a tendency to decrease the calcium content in the oral fluid with the development of inflammatory-dystrophic process in the periodontium, on the contrary - phosphorus levels have increased.

Negative dynamics of calcium-phosphorus molar coefficient was found in patients with hypothy-

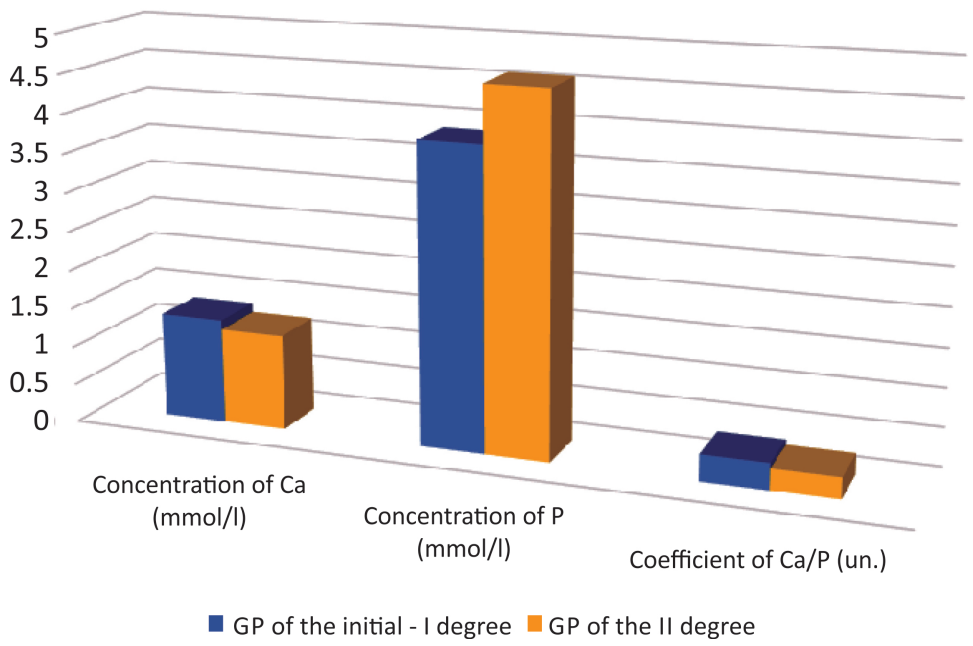

FIGURE 1. Indices of calcium/phosphorus homeostasis of the oral fluid in patients of the group I 


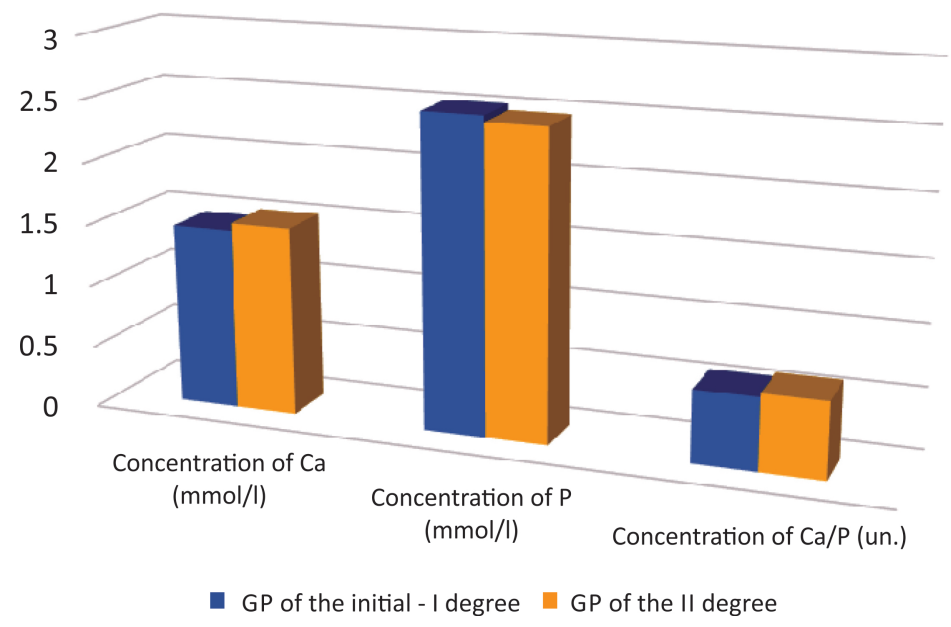

FIGURE 2. Indices of calcium/phosphorus homeostasis of the oral fluid in patients of the group II

roidism. The results of studies indicate a problem in the absorption of calcium in patients with hypothyroidism. This suggests that for normal absorption and metabolism of calcium thyroid hormones are required, the production of which is reduced in this endocrine pathology. The data of our research are consistent with the conclusions of other authors having studied this topic [13-15]. According to Cardoso LF et al., the bone remodeling process is an ingenious mechanism that simultaneously contributes to calcium homeostasis and bone resistance. Under ideal conditions, the final product of bone remodeling is the maintenance of bone integrity. Conversely, imbalance in bone formation and resorption leads to bone loss and deterioration of bone microarchitecture, with consequent emergence of bone fragility and an increased risk of fractures. Hypothyroidism slows down bone metabolism, favoring resorption and a negative calcium balance and as a result bone loss [16]. In attempts to elucidate factors stimulating bone resorption in pa- tients with different inflammatory diseases in the vicinity of the skeleton, peridontal disease, authors Rasmussen et al. are investigating the presence of bone-resorbing activity in a variety of inflammatory exudates [17]. We agree with other authors that hypothyroidism causes negative changes in bone mineral composition and complicates the course of periodontal disease [18-23].

\section{CONCLUSIONS}

Thus, the analysis of research results shows that in young people with periodontal diseases and hypothyroidism there is a pathological change in metabolic processes, increased excretion of mineral components, especially calcium. Periodontal pathology against the background of hypothyroidism is characterized by the development of chronic inflammatory process together with a pronounced decrease in calcium-phosphorus homeostasis of the oral fluid.

Conflict of interest: none declared Financial support: none declared

\section{REFERENCES}

1. Zelinska NB, Larin OS. Pathology of thyroid gland in the children's population of Ukraine]. Clinical Endocrinology and Endocrine Surgery. 2016;3(55):76-81.

2. Tymkiv VV. Statistical analysis of non-tumor diseases of the thyroid gland in Ivano-Frankivsk region during 2007-2011. Galician Medical Journal. 2014;21(1):96-100.

3. Bridwell RE, Willis GC, Gottlieb M, Koyfman A, Long B. Decompensated hypothyroidism: A review for the emergency clinician. Am J Emerg Med. 2021;39:207-12.

4. Aldulaijan HA, Cohen RE, Stellrecht EM, Levine MJ, Yerke LM. Relationship between hypothyroidism and periodontitis: A scoping review. Clin Exp Dent Res. 2020;6(1):147-57.

5. Shcherba VV, Korda MM. The role of thyroid gland dysfunction in the pathogenesis of generalized periodontitis (literature review). Clin Dent. 2018;1:60-70.

6. Kwon M, Jeong YJ, Kwak J, Jung KY, Baek SK. Association between oral health and thyroid disorders: A population-based cross-sectional study. Oral Dis. 2021;30.

7. Patil BS, Patil S, Gururaj TR.Niger. Probable autoimmune causal relationship between periodontitis and Hashimotos thyroiditis: a systemic review. J Clin Pract. 2011;14(3):253-61.

8. Morais A, Resende M, Pereira J. Hashimoto Thyroiditis and Periodontal Disease: A Narrative Review. Acta Med Port. 2016;29(10): 651-7. 
9. Piksin IN, Davyidkin VI, Moskovchenko AS, Vilkov AV, Kechaykin AN. The state of bone metabolism in diseases of the thyroid gland. Medical Almanac. 2016;4(44):154-7.

10. Tuchendler $D$, Bolanowski $M$. The influence of thyroid dysfunction on bone metabolism. Thyroid Research. 2014;7:12.

11. Ziablitskaya MS, Atrushkevich VG. Pathogenetic bases of the generalized periodontitis development against the background of calcium and vitamin D metabolism disorders. Innovations in Dentistry. 2014;1:2-28

12. Martina E, Campanati A, Diotallevi F, Offidani A. Saliva and Oral Diseases. J Clin Med. 2020;9(2):466.

13. Zorina OA, Boriskina OA, Magomedov RN, Prohodnaya VA, Mironycheva KV, Vasil'ev AV. Dynamics of bone metabolism markers in oral fluid after surgical treatment of chronic periodontal disease with various osteoplastic materials. Stomatologiia (Mosk). 2019; 98(2):27-30.

14. Uspenskaya OA, Kachesova ES, Abasnia SR, Shevchenko EA, Fadeeva II. Metabolic bone tissue markers dynamics as criteria for periodontal disease treatment efficiency. Stomatologiia (Mosk). 2021;100(2):4449.

15. Beriashvili S, Nikolaishvili M, Mantskava M, Momtsemlidze N, Franchuk K. Changes In Tooth Hard Tissue Minerali-Zation And Blood Rheology In Healthy Adolescents And Those With Thyroid Dysfunction. Georgian Med News. 2016 Nov:28-34.
16. Cardoso LF, Maciel LM, Paula FJ.The multiple effects of thyroid disorders on bone and mineral metabolism. Arq Bras Endocrinol Metabol. 2014 Jul;58(5):452-63.

17. Rasmussen L, Hänström L, Lerner UH. Characterization of bone resorbing activity in gingival crevicular fluid from patients with periodontitis. Clin Periodontol. 2000 Jan;27(1):41-52.

18. Williams GR, Bassett JHD.Thyroid diseases and bone health. J Endocrinol Invest. 2018 Jan;41(1):99-109.

19. Rayman MP. Multiple nutritional factors and thyroid disease, with particular reference to autoimmune thyroid disease. Proc Nutr Soc. 2019 Feb;78(1):34-44.

20. Talebi S, Ghaedi E, Sadeghi E, Mohammadi H, Hadi A, Clark CCT, Askari G.Trace Element Status and Hypothyroidism: A Systematic Review and Meta-analysis. Biol Trace Elem Res. 2020 Sep;197(1):1-14.

21. Kawicka A, Regulska-llow B. Metabolic disorders and nutritional status in autoimmune thyroid diseases. Postepy Hig Med Dosw. $2015 \mathrm{Jan}$ 2;69:80-90.

22. Chang CY, Rosenthal DI, Mitchell DM, Handa A, Kattapuram SV, Huang AJ.Imaging Findings of Metabolic Bone Disease. Radiographics. 2016 Oct;36(6):1871-1887.

23. Apostu D, Lucaciu O, Oltean-Dan D, Mureșan AD, Moisescu-Pop C, Maxim A, Benea H.The Influence of Thyroid Pathology on Osteoporosis and Fracture Risk: A Review. Diagnostics (Basel). 2020 Mar 7;10(3):149. 